\title{
Study of Thermal Properties on UV-Curable Coatings Derived from Oleochemical Polyols
}

\author{
MEHUL M. PATEL ${ }^{*}$, CHIRAG J. PATEL and NATVAR K. PATEL \\ *Gujarat Industrial Research and Development Agency, Vadodara-390002, Gujarat, India \\ Department of Chemistry, Sardar Patel University, Vallabh Vidyanagar, Gujarat, India \\ meh_chem@yahoo.com
}

Received 13 May 2012 / Accepted 30 May 2012

\begin{abstract}
Over the last few years, UV radiation curing has become a well established coating technology used for several different applications. Polymeric component based on renewable resource is another area for getting low VOC coatings. In the present study cellulose and soya fatty acid were used to prepare oleochemical polyols which in turn reacted with diisocyanates to get PU which is further reacted with hydroxyl terminated acrylates to get oligomeric urethane acrylates. These polymeric compounds are used without any organic solvent for UV-coating formulation and cured by UV light on the metal surfaces. Thus effect of different types of polyols were used to prepared UV coatings studied on thermal properties like dynamic mechanical properties (DMA) and thermogravimetric analysis (TGA)
\end{abstract}

Keywords: UV Radiation, Oleochemical Polyol, Dynamic mechanical properties (DMA), Thermogravimetric analysis (TGA)

\section{Introduction}

UV-radiation curable coatings represent a class of coatings with no or little volatile organic compounds (VOCs). In addition, compared to thermally cured coatings, the use of UVradiation curable coatings offers some advantages, such as instant drying, broad formulating range, reduced energy consumption, coating of heat sensitive substrate, and low space and capital requirement for curing equipment ${ }^{1}$. UV radiation curing is a technique widely used to prepare transparent coatings with good properties. The widespread interest for UV systems can be explained by several reasons such as their rapid polymerization, which can be controlled by light intensity, and their very low energy consumption. Moreover, UV curing technology allows low temperature operation, application versatility and good viscosity control. This technology is also very attractive from the environmental point of view since no organic solvents are required. The greatest advantage of UV coatings comes from the general properties improvement. Polymers cured by UV light usually exhibit superior 
mechanical, chemical and heat resistance because of their higher cross-linking density ${ }^{2-3}$. Among the oligomers used for UV-curable coatings, polyurethane acrylate (PUA) oligomers have gained more and more attention and speedy development due to a wide range of excellent application properties, such as high impact and tensile strength, abrasion resistance and toughness combined with excellent resistance to chemicals and solvents ${ }^{4-7}$. UV-curable coating resins basically include a prepolymer, one or several reactive diluents, a photoinitiator and other additives in minute amounts (surfactants, stabilizers, etc. $)^{8}$.

In the present research work, soya epoxy ester were prepared by soya fatty acid and epoxy resin. And these soya epoxy ester were reacted with different hydroxyl containing polyols like ethylene glycol, trimethylol propane, pentaerythritol and different cellulose based derivatives have been used to prepare oleochemical polyols. Further these oleochemical polyols were converted to urethanes prepolymer by reacting -NCO groups of TDI and IPDI. Then, these urethanes were converted to urethane acrylate oligomer by reacting - NCO end group of urethane with HEMA. UV curable coating compositions were obtained by blending these oligomers with reactive diluents (cross-linking monomer), photoinitiator and other additives. These compositions were cured under UV light at (200 Watt/inch) (280-360 nm) medium pressure mercury vapor lamp of UV-2KW-2-35 intensity. These coating were evaluated to study on dynamical mechanical analysis (DMA) and thermogravimetric analysis (TGA) effect of different polyols and isocyante content.

\section{Experimental}

Soya Epoxy ester were procured from M/s. Champion Industries, Vallabh Vidyanagar. Its characteristic details are given in Table 1. Cellulose, used was extracted from bagasse and purified in our laboratory using well-known nitric acid process and this cellulose were used to prepared cellulose glycoglycoside. This glycoglycoside were prepared and characterized by our recent reported work 9 .

Table 1. Characterization of soya epoxy ester

\begin{tabular}{|c|c|c|c|c|c|c|}
\hline Code & $\begin{array}{l}\text { \% Composition } \\
\text { Acid : Epoxy }\end{array}$ & g./L. & $\begin{array}{c}\% \\
\text { NVM }\end{array}$ & Colour & $\begin{array}{l}\text { Viscosity } \\
\text { Cst }\end{array}$ & $\begin{array}{c}\text { Acid Value } \\
\mathrm{mg} \mathrm{of} \mathrm{KOH/g} \text { of } \\
\text { sample }\end{array}$ \\
\hline
\end{tabular}

$\begin{array}{lllllll}\text { ES } & 0.4: 1 & 0.93 & 99 & 5 & 180-200 & 4-7\end{array}$

Ethylene glycol, propylene glycol, trimethylol propane and pentaerythritol, sulfuric acid, triethyl amine was procured from Chitichem Corporation, Baroda. TDI and IPDI were received from Grand polycoats, Vadodara, India

\section{Method}

\section{Synthesis of oleochemical polyols}

The oleochemical polyols were synthesized by reacting epoxy ester (ES) and ethylene glycol, trimethylol propane, pentaerythritol and different cellulose glycoglycoside in three necked flask equipped with nitrogen inlet and water condenser in presence of triethyl amine (TEA) catalyst. The reaction was carried out at reflux temperature for $3 \mathrm{~h}$. The epoxy value was determined from time to time until the epoxy value was almost zero. The formulations used for polyol preparation are shown in Table 2. The characterization of hyperbranched polyol are shown in Table 3. 
Table 2. Preparation of oleochemical polyol

\begin{tabular}{|c|c|c|c|c|c|c|}
\hline $\begin{array}{l}\text { S. } \\
\text { No. }\end{array}$ & $\begin{array}{l}\text { Name of } \\
\text { Chemical }\end{array}$ & $\begin{array}{c}\text { Set-I } \\
\text { (ESG) } \\
\text { Wt. in g }\end{array}$ & $\begin{array}{c}\text { Set-II } \\
\text { (EST) } \\
\text { Wt. in g } \\
\end{array}$ & $\begin{array}{c}\text { Set-III } \\
\text { (ESP) } \\
\text { Wt. in g }\end{array}$ & $\begin{array}{l}\text { Set-IV } \\
\left(\text { ESC }_{1}\right) \\
\text { Wt. in } g\end{array}$ & $\begin{array}{c}\text { Set-V } \\
\left(\mathrm{ESC}_{2}\right) \\
\text { Wt. in } \mathrm{g}\end{array}$ \\
\hline 1 & ES & 47.18 & 43.90 & 43.82 & 37.00 & 35.75 \\
\hline 2 & EG & 2.81 & - & - & - & - \\
\hline 3 & TMP & - & 5.66 & - & - & - \\
\hline 4 & PENTA & - & - & 5.73 & - & - \\
\hline 5 & $\mathrm{C}_{1}$ & - & - & - & 12.50 & - \\
\hline 6 & $\mathrm{C}_{2}$ & - & - & - & - & 14.00 \\
\hline 7 & TEA & 0.47 & 0.43 & 0.43 & 0.37 & 0.35 \\
\hline
\end{tabular}

EG : Ethylene Glycol, TMP : Trimethylol Propane, PENTA: Pentaerythritol, $C_{1}:$ Glycoglycoside with ethylene glycol, $C_{2}$ : Glycoglycoside with propylene glycol

Table 3. Characterization of hyperbranched oleochemical polyol

\begin{tabular}{lccccc}
\hline \multicolumn{1}{c}{ Code } & Colour & g/L. & \% NVM & Hydroxyl Value & Mol. Wt. \\
\hline ESG & 6 & 1.10 & 99.32 & 300.10 & 655 \\
EST & 6 & 1.22 & 99.25 & 354.02 & 758 \\
ESP & 7 & 1.28 & 99.36 & 441.02 & 775 \\
ESC $_{1}$ & 7 & 1.25 & 99.54 & 395.00 & 852 \\
ESC $_{2}$ & 8 & 1.29 & 99.60 & 372.34 & 904 \\
\hline
\end{tabular}

Preparation of urethane acrylate

A dry, four necked-flask was placed in a water bath, polyisocyanates (TDI/IPDI) added and temperature of the bath raised to 50-55 ${ }^{\circ} \mathrm{C}$. HEMA was added drop wise to the reaction flask through dropping funnel (within $2 \mathrm{~h}$ ). Temperature of bath was controlled within a $50-55^{\circ} \mathrm{C}$ and reaction allowed to continue until desired percentage of -NCO obtained. Oleochemical polyols was added drop wise through dropping funnel at the same reaction condition and allowed to continue until desired percentage of - NCO acquired. The reaction progress was monitored by determining unreacted $-\mathrm{NCO}$ groups by dibutylamine back titration method ${ }^{10}$. The reaction was allowed to continue until the percentage of - $\mathrm{NCO}$ became zero. The resultant urethane acrylate oligomer was found transparent viscous liquid. These, prepared urethane acrylate oligomer were subsequently used for the preparation of UV curable coating composition.

\section{Preparation of UV curable coating composition}

The urethane acrylate oligomer was taken in a beaker and after addition of stoichiometric amount of reactive diluents, photoinitiator the contents were stirred at room temperature.

\section{Application and curing of coating composition}

These films were prepared by spraying the coating onto silicon release paper. The coating was exposed to a (200 Watt/inch) $(280-360 \mathrm{~nm})$ medium pressure mercury vapor lamp of UV-2KW-2-35 curing system for 1-2 min after separating them from the release paper. Data were normalized according to individual sample dimensions.

\section{Measurements}

The dynamic mechanical properties were obtained using DMA analyzer Triton Technology Ltd, U.K. Tritec 2000 model. The testing was carried out in a three point flexural mode. The specimen was heated at rate of $10{ }^{\circ} \mathrm{C} / \mathrm{min}$. The frequency used was $1 \mathrm{~Hz}$. The storage modulas (E') and the loss tangent (tan delta) were obtained as a function of temperature. 
The glass transition temperature $(\mathrm{Tg})$ of the films was obtained from the peaks of the loss tangent (tan delta) curve. The thermogravimetric properties were obtained using STA 449 F3 Jupiter, Netzsch, German at RT to $700{ }^{\circ} \mathrm{C}$ on heated at rate of $10{ }^{\circ} \mathrm{C} / \mathrm{min}$.

\section{Results and Discussion}

Dynamic mechanical analysis (DMA)

UV cured polyurethane acrylate films possess a two phase microstructures one of the diisocyanate and flexible long chain diols soft segment. The microstructure of UV cured polyurethane acrylates were investigated thought the measurement of dynamic mechanical properties. The effect of the structure of urethane acrylate for three different types and different functionality of the polyols were investigated. The storage modulus(E'), loss modulus(E") and loss factor(tan $\delta$ ) obtained at $1 \mathrm{~Hz}$ are shown in Figures 1-3. The dynamic mechanical properties of the UV cured films for sample (EST, ESP, ESC1 and ESC2) were determined as a function of temperature over the temperature range of $0{ }^{\circ} \mathrm{C}$ to $250{ }^{\circ} \mathrm{C}$.

Loss modulus (E") vs. temperature of the UV cured films is shown in Figure 1. In the sets of ESC1 and ESC2 has higher area of curve for loss modulus comparison with other set of the sample like EST and ESP. This result due to the increase the molecular weight of the polyurethane acrylate affects the loss modulus (E") and functionality of oleochemical polyols affect of the temperature of loss modulus (E").

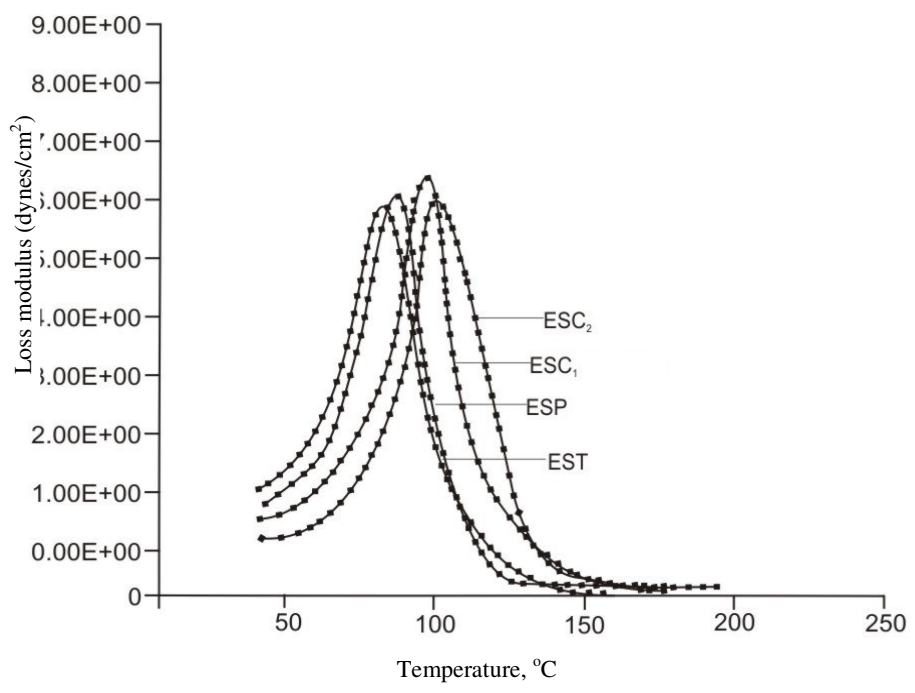

Figure 1. Loss modulus vs. temperature

Storage modulus $v s$. temperature of the UV cured films is plotted in Figure 2. The molecular weight of different oleochemical polyols increases with increase the value of storage modulus (E') as shown in Figure 2. The higher functionality of oleochemical polyol like ESC1 and ESC2 is also increased with the increased the storage modulus (E') value compared to EST, ESP. This result may be due to the increase the molecular weight and functionality of oleochemical polyols of the polyurethane acrylate also affects the storage modulus (E'). Loss factor $(\tan \delta) v s$. temperature of the UV cured films were plotted in Figure 3. In the sets of ESC1 and ESC2 has higher value of the glass transition temperature comparison with other set of the sample like EST, ESP. 


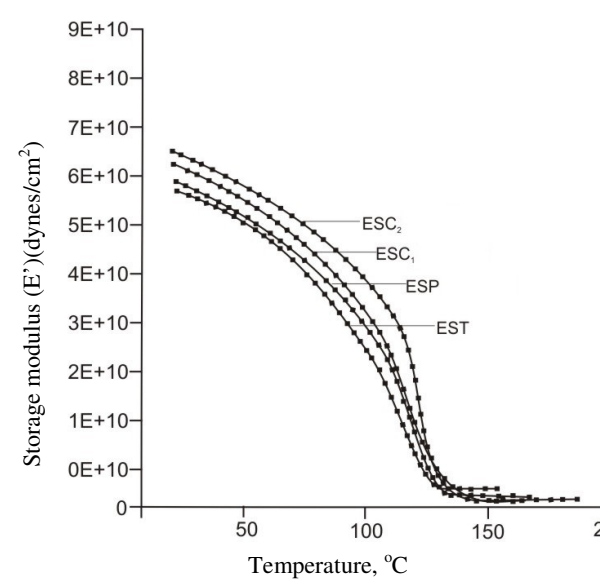

Figure 2. Storage modulus $v s$. temperature

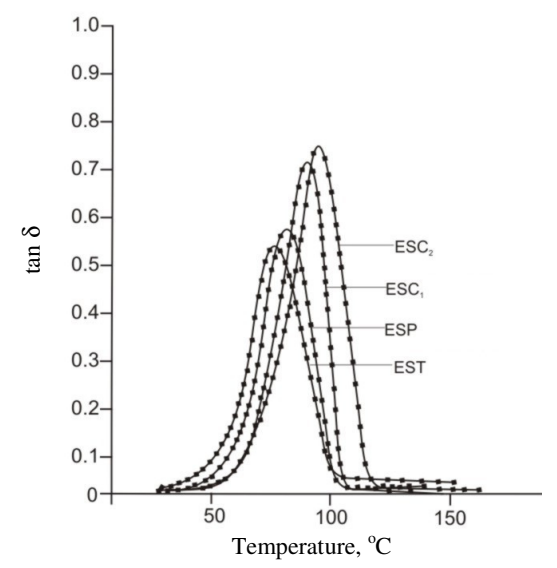

Figure 3. Loss $\tan \delta v s$. temperature

The $\tan \delta$ indicated that the ESC1 and ESC2 have the highest glass transition temperature as well as the largest peak among the other set samples this due to the increased the molecular weight of the polyurethane acrylate cured film increased the glass transition value and functionality of oleochemical polyols affect the loss factor $(\tan \delta)$ value.

The softening point $(T \mathrm{~s})$ is defined as the extrapolated onset of the drop of log $\left(E^{\prime}\right)$. The glass transition temperature $(T \mathrm{~g})$ is defined as the peak position of a $\tan (\delta)$ curve. The $T \mathrm{~s} / T \mathrm{~g}$ ratio expresses the width of a $\tan (\delta)$ peak, it is a rule that a higher $T \mathrm{~s} / T \mathrm{~g}$ ratio leads to a narrow $\tan (\delta)$ peak and the obtained film is more homogeneous. The dynamic mechanical thermal properties of these UV-cured films are shown in Figure 1-3. It has been observed that the ESC1 and ESC2 based UV-Polyurethane coating has the highest $T \mathrm{~s}$ and $T \mathrm{~g}$ compare to EST \&ESP because of its highest crosslink density, due to higher molecular weight and higher functionality molecular structure. Moreover, from the data in Table 4.

Table 4. Data from DMA for the UV-cured film

\begin{tabular}{cccc}
\hline Sample & $\mathrm{Ts},{ }^{0} \mathrm{C}$ & $\mathrm{Tg},{ }^{\circ} \mathrm{C}$ & $\mathrm{Ts} / \mathrm{Tg}$ \\
\hline $\mathrm{EST}$ & 62 & 73 & 0.849 \\
$\mathrm{ESP}$ & 68 & 84 & 0.809 \\
$\mathrm{ESC}_{1}$ & 83 & 94 & 0.882 \\
$\mathrm{ESC}_{2}$ & 90 & 99 & 0.909 \\
\hline
\end{tabular}

Thermogravimetric analysis (TGA)

The TGA thermogram in Figure $4 \& 5$ and thermogram data in Table $5 \& 6$ are clearly indicate good thermal stability of cured film. Data indicated by negligible weight loss shows that all the UV coating films are stable up to $150{ }^{\circ} \mathrm{C}-250{ }^{\circ} \mathrm{C}$ irrespective of types and amount of polyol and urethane linkage. Thermograms indicate that all the UV coating films degraded as not well-distinguished three stages. The degradation in the first stage of polyurethanes may correspond to the breaking of urethane bond and leading the formation of $\mathrm{CO}_{2}$, alcohols, amines carbon monoxide etc. 


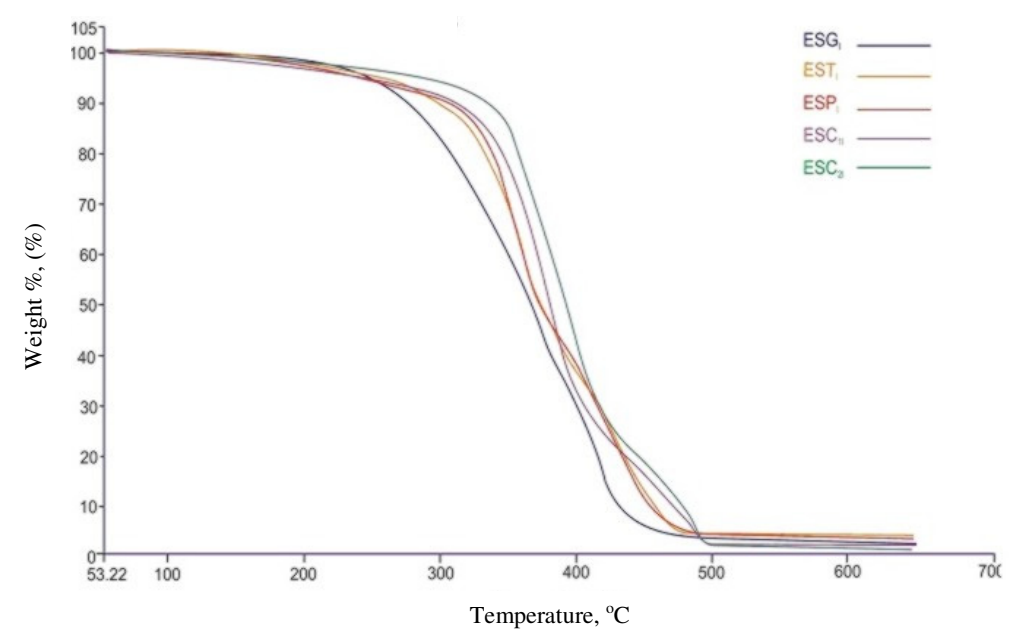

Figure 4. Thermogravimetry analysis of UV cured film (Aromatic)

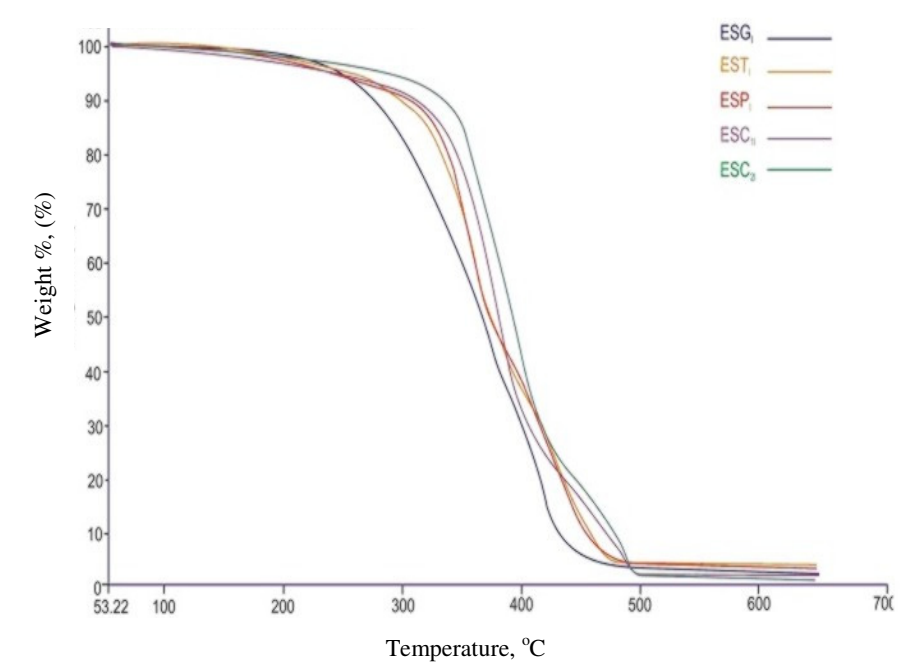

Figure 5. Thermogravimetry analysis of UV cured film (Aliphatic)

Table 5. TGA data of UV cured film-Aromatic

\begin{tabular}{|c|c|c|c|c|c|c|}
\hline \multirow[t]{2}{*}{ Code } & \multicolumn{3}{|c|}{$\begin{array}{c}\% \text { Weight loss at different } \\
\text { temperature, }{ }^{0} \mathrm{C}\end{array}$} & \multirow{2}{*}{$\begin{array}{l}\text { Decomposition } \\
\text { range, }{ }^{0} \mathrm{C}\end{array}$} & \multirow{2}{*}{ IPDT, ${ }^{0} \mathrm{C}$} & \multirow{2}{*}{$\begin{array}{l}\text { Activation } \\
\text { energy }\left(\mathrm{E}_{\mathrm{A}}\right) \\
\left(\mathrm{k} . J . \mathrm{mole}^{-1}\right)\end{array}$} \\
\hline & 250 & 350 & 450 & & & \\
\hline $\mathrm{ESG}_{\mathrm{T}}$ & 4.98 & 60.45 & 94.57 & $220-455$ & 327 & 32.68 \\
\hline $\mathrm{EST}_{\mathrm{T}}$ & 5.25 & 56.34 & 95.86 & $229-469$ & 336 & 34.27 \\
\hline $\mathrm{ESP}_{\mathrm{T}}$ & 5.56 & 48.34 & 92.70 & $230-477$ & 353 & 35.83 \\
\hline $\mathrm{ESC}_{1 \mathrm{~T}}$ & 3.77 & 36.26 & 74.82 & $247-515$ & 360 & 38.96 \\
\hline $\mathrm{ESC}_{2 \mathrm{~T}}$ & 2.83 & 38.48 & 81.72 & $255-519$ & 370 & 42.37 \\
\hline
\end{tabular}


Table 6. TGA data of UV cured film-Aliphatic

\begin{tabular}{lcccccc}
\hline \multirow{2}{*}{ Code } & \multicolumn{3}{c}{$\begin{array}{c}\text { Weight loss at different } \\
\text { temperature, }{ }^{0} \mathrm{C}\end{array}$} & $\begin{array}{c}\text { Decomposition } \\
\text { range, }{ }^{0} \mathrm{C}\end{array}$ & IPDT, ${ }^{0} \mathrm{C}$ & $\begin{array}{c}\text { Activation } \\
\text { energy }\left(\mathrm{E}_{\mathrm{A}}\right) \\
\left(\mathrm{k} . J . ~ m o l e^{-1}\right)\end{array}$ \\
\cline { 2 - 4 } & 250 & 350 & 450 & & & 35.74 \\
$\mathrm{ESG}_{\mathrm{I}}$ & 7.62 & 46.87 & 95.53 & $210-456$ & 360 & 38.27 \\
$\mathrm{EST}_{\mathrm{I}}$ & 7.39 & 47.73 & 90.03 & $212-475$ & 365 & 41.03 \\
$\mathrm{ESP}_{\mathrm{I}}$ & 7.13 & 40.37 & 92.75 & $260-481$ & 369 & 43.84 \\
$\mathrm{ESC}_{1 \mathrm{I}}$ & 7.28 & 31.72 & 86.15 & $268-490$ & 377 & 45.17 \\
$\mathrm{ESG}_{2 \mathrm{I}}$ & 2.75 & 16.36 & 80.50 & $270-500$ & 380 & \\
\hline
\end{tabular}

Second stage at about $250-350{ }^{\circ} \mathrm{C}$ may be due to the decomposition of ether and ester linkage. In the case of UV coating films from different types of polyol, the main chain degradation may occur with the formation of as evidenced by the thermal degradation of fatty acid. In the third step finally this cured product degrades completely at slow rate. The percentage of weight loss up to $250{ }^{\circ} \mathrm{C}$ in aromatic isocyanate based UV coating film is slightly higher compared to aliphatic isocyanate based UV coating films because of the instability of aliphatic moiety and increase the cross linking density the percentage of weight loss decreases.

The activation energy of all the UV coating film are in the range of 30 to $45 \mathrm{~kJ} / \mathrm{mole}$, the UV curable polyurethaneacrylate from cellulose based oleochemical polyols (ESC1 and ESC2) having high stability (i.e. having higher activation energy) compared to ESG, EST and ESP based oleochemical polyols. Because of cellulose has high unsaturation; high molecular weight, crosslink density compared to other based UV coating films.

\section{Conclusion}

A series of urethane acrylates derived from different hydroxyl group containing oleochemical polyols was prepared in simple, two-stage process. These materials were used as oligomers in radiation curable compositions. Soya epoxy ester was able to obtain very low viscosity and at the same time a high functionality urethane acrylate resin. Coatings obtained from these oligomers were characterized by dynamical mechanical analysis, thermogravimetric analysis, The UV cured samples from cellulose based oleochemical polyols exhibit good mechanical and thermal properties, their mechanical properties are more dependent on double bonds from unsaturated contribute to curing with extra cross-linking by an oxidative mechanism. It leads to a formation of new cross-links and additional improvement in the properties of the previously UV cured samples.

\section{Acknowledgement}

Authors are thankful to Dr. N. K. Patel, Professor of the Chemistry Department, Sardar Patel University, for providing necessary laboratory facilities.

\section{Reference}

1. Dzunuzovic E, Tasic S, Bozic B, Babic D and Dunjic B, Progr Org Coat., 2005, 52, 136-143.

2. Bauer F, Sauerland V, Glasel H-J, Ernst H, Findeisen M, Hartmann E, Langguth H, Marquart B and Mehnert R, Macromol Mater Eng., 2002, 287, 8, 546-552. 
3. Bauer F, Glasel H-J, Decker U, Ernst Freyer A, Hartmann E, Sauerland V and Mehnert R, Prog Org Coat., 2003, 47(2), 147-153.

4. Bai C Y, Zhang X Y, Dai J B, Li W H, Progr Org Coat., 2006, 55(3), 291-295.

5. Xu J, Pang W and Shi W, Thin Solid Films, 2006, 514(1-2), 69-75.

6. Lu W H, Xu W J, Wu Y M, Zhou X, Lu Y B and Xiong Y Q, Progr Org Coat., 2006, 56, 252-255.

7. Park M-H, Jang W, Yang S J, Shul Y and Han H, J Appl Polym Sci., 2006, 100, 113-123.

8. Pappas S P, Radiation Curing, Science and Technology, Plenum Press, New York and London, 1992.

9. Patel MM, Patel K I, Patel H B and Parmar J S, Iranian Polym J., 2009, 18(11), 903-915.

10. Ottey F H, Zagoren B L and Mehltretter C L, I \& EC Product Res Dev., 1965, 4(4), 228. 